\title{
An On-line Automatic Aluminum Wheel Recognition System
}

\author{
Yiyang Zhang ${ }^{1, ~ a}$, Shuangyong $\mathrm{Liu}^{2, \mathrm{~b}}$, Shaobo Sun ${ }^{3, \mathrm{c}}$, Yong Han ${ }^{4, \mathrm{~d}}$ \\ ${ }^{1,3}$ School of Material Science and Engineering, Beihang University, Beijing 100191, China \\ ${ }^{2,4}$ CITIC Dicastal Co., Ltd., Qinhuangdao 066011, China \\ azhangyiyang@buaa.edu.cn, bliushuangyong@dicastal.com, \\ csunshaobo@buaa.edu.cn, dhanyong@dicastal.com
}

Keywords: aluminum wheel, automatic recognition system, laser measuring.

\begin{abstract}
Identifying various types of aluminum wheels was traditionally performed manually, which could result in low efficiency, limited reliability, poor accuracy, and high labor cost. This paper presents the design and implementation of an on-line automatic recognition system for aluminum wheels based on laser trigonometry principle. The system mainly consists of a recognition station, two laser displacement sensors, a ball-screw, an industrial computer with data processing software, and a programmable logic controller (PLC). Robust algorithms, as well as an ingenious database for storing information of wheels in different styles and size are introduced for identifying the wheels. The feature data of a special wheel, such as the diameter, thickness and number of spokes, can be determining accurately by scanning the wheel using two laser displacement sensors and compare well with those in the database. Results show that the system identifies each wheel correctly in less than 20 seconds. The stability of this system is excellent. Significant cost saving, low error rate, and high efficiency can be achieved.
\end{abstract}

\section{Introduction}

With the automation and intelligence of car production, the automobile manufacturing capacity is booming, which has led to a quite strong competition in the world. In order to use less materials and improve the performance of automobiles, the aluminum wheels have been widely utilized in automobiles due to its quick heat dispelling, less defects, light weight, and elegant appearance since the $21^{\text {st }}$ century ${ }^{[1]}$.During the last 6 consecutive years, the numbers of cars' production and sales in China have always ranked first in the world ${ }^{[2]}$.As the sales and production of automobiles increases, the demand of aluminum wheels with various types and sizes is exploding. In actual production, different types of casted aluminum wheels are inspected by X-ray and heat-treated on a same production line. Then each wheel on the roller bed should be identified and classified correctly and efficiently for further special machining procedures.

Fig. 1 shows a typical aluminum wheel. Different type of wheel usually has different combination of the diameter, thickness, number and size of spokes. According to this characteristic information, the wheel can be identified.

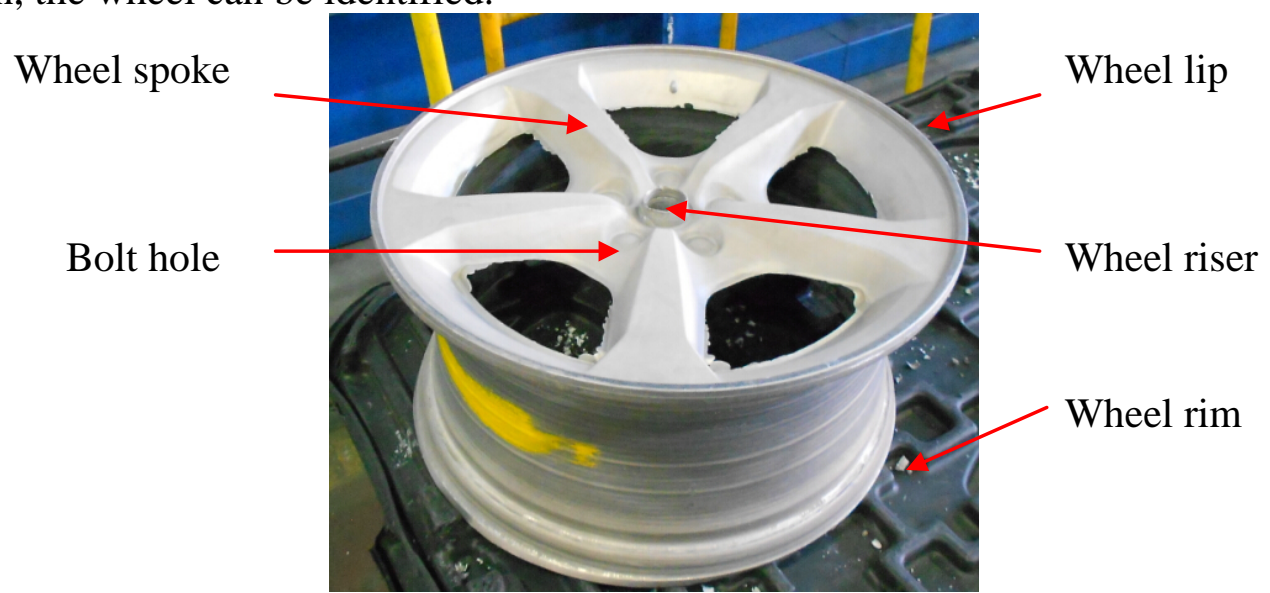

Fig.1 The typical aluminum wheel 
Traditionally, the aluminum wheels identification is based on the operators' observation, which could result in limited reliability, low accuracy, high-labor cost, and time consuming. In terms of the above considerations, it is necessary to develop a new method to identify wheels.

The present paper proposes an on-line recognition system for aluminum wheels based on laser trigonometry principle, which is capable of identifying wheels automatically. This system incorporates technologies of optics, mechanics, and electronics, which can overcome the disadvantages of manual method. The design and implement of the automatic wheel recognition system will be presented in detail in the following sections.

\section{Measurement principle and recognition method of the system}

\section{Measurement principle of the laser trigonometry.}

The recognition system is based on the laser trigonometry, which is one of the photoelectric detection technologies. By using laser trigonometry people can measure the displacement, vibration and thickness of an object in real time ${ }^{[3]}$. Since the laser trigonometry has many advantages, such as simple structure, fast measurement speed, strong processing ability in real time, flexible and convenient use, it has been widely used in industry ${ }^{[4][5]}$.

As shown in Fig.2, the laser beam is projected onto an object, and then the reflected laser point will be imagined on a detector, which is called diffuse reflection. If the object changes its position from point 1 to point 2, the image on the detector changes its original position accordingly from point $1^{\prime}$ to point $2^{\prime}$. In the diagram, $s$ is the object distance; $s^{\prime}$ is the image distance; $d$ is the displacement of image point; and $\delta$ is the actual displacement of the object. With the relationship between the displacement of the image point and the actual displacement of the object, the displacement of the object can be calculated. In order to implement focusing perfectly, the optical system should meet Scheimpflug Condition, that an image plane, an object plane and a principle plane of the lens should meet in one point, as point $x$ shown in Fig. ${ }^{[4][6]}$.

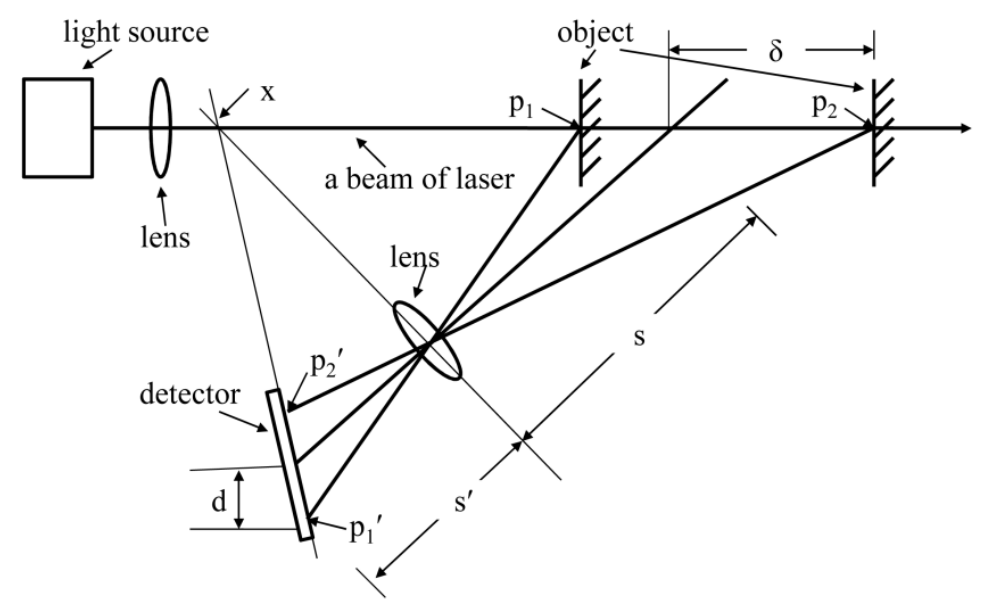

\section{Recognition system structure.}

Fig.2 The principle of laser triangulation

Fig.3 shows the schematic diagram of the automatic aluminum wheel recognition system. The system comprises a recognition station for centering, lifting and turning the wheel, a roller bed for delivering the wheel, two laser displacement sensors used to scan the wheel, a ball-screw and a servo motor used to drive laser displacement sensors, an industrial computer with robust software used to calculate the collected data, a programmable logic controller (PLC) interpreting and initiating the signal for the system, and a database storing feature data of various types of wheels. 


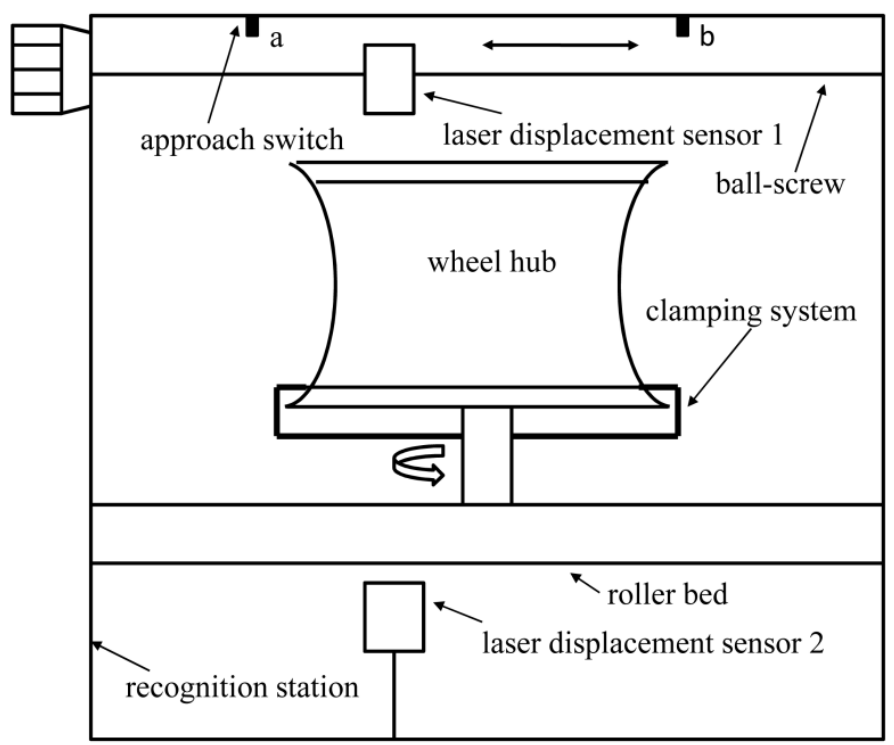

Fig.3 The structure of the wheel recognition system

During the recognition process, when a wheel is delivered to the recognition station by the roller bed, a signal is sent to PLC to control the recognition station to clamp the wheel. As the wheel being adjusted centering and lifted, PLC generate a signal to the industrial computer, and then the industrial computer control laser displacement sensor 1 driven by a ball-screw to scan the wheel at a uniform velocity of $250 \mathrm{~mm} / \mathrm{s}$ along the centerline of the wheel from point a to point $\mathrm{b}$. As long as laser displacement sensor 1 reaches point b, it stops scanning and initiates a signal to PLC which will stop the movement of laser displacement sensor 1. Then, PLC controls the station to rotate the wheel. Laser displacement sensor 2 then scans the spokes while the wheel is rotated. It is worth noting that the height of laser displacement sensor 2 can be adjusted automatically to meet the measurement of various wheels. When one rotation is finished, the movement of the wheel and laser scanning stops. After the above procedures, the laser displacement sensors and wheel recognition station return to the initial state. The data collected by the two laser displacement sensors are transferred to the industrial computer through universal serial bus (USB) communication. The scanning strategy of the two laser displacement sensors is shown in Fig.4.
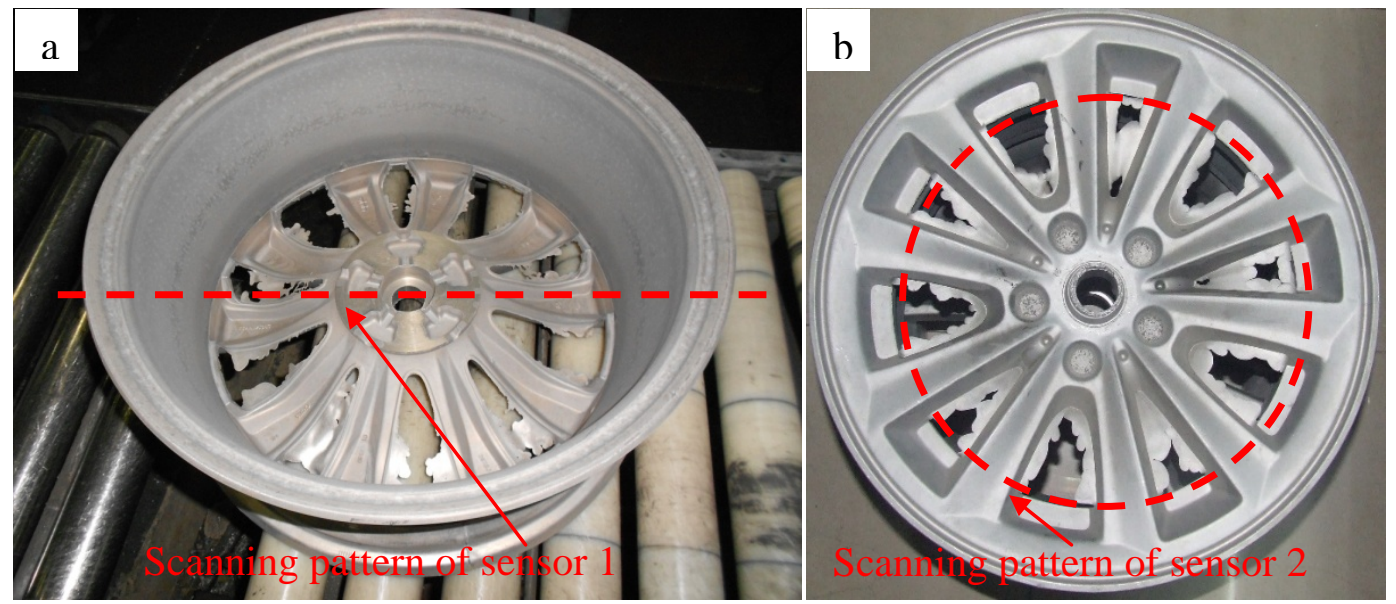

Fig.4 The scanning strategy of the two laser sensors

\section{Computing method.}

The diameter, thickness, and number of spokes which can be calculated by data points scanned by the laser displacement sensors are selected to be the identifying information for wheel 
recognition in this system. Once, the data points' collecting of the sensors is finished, these points will be transferred into the industrial computer immediately by USB communication.

Fig. 5 shows the typical wheel outline scanned by the laser displacement sensor 1, plotting the number and relative height of each point. With the relationship between these points and the sample-rate, the diameter can be calculated by the special computer soft ware. Furthermore, the thickness can be obtained when the relative heights of the wheel and the recognition station are obtained by the sensors' scanning.

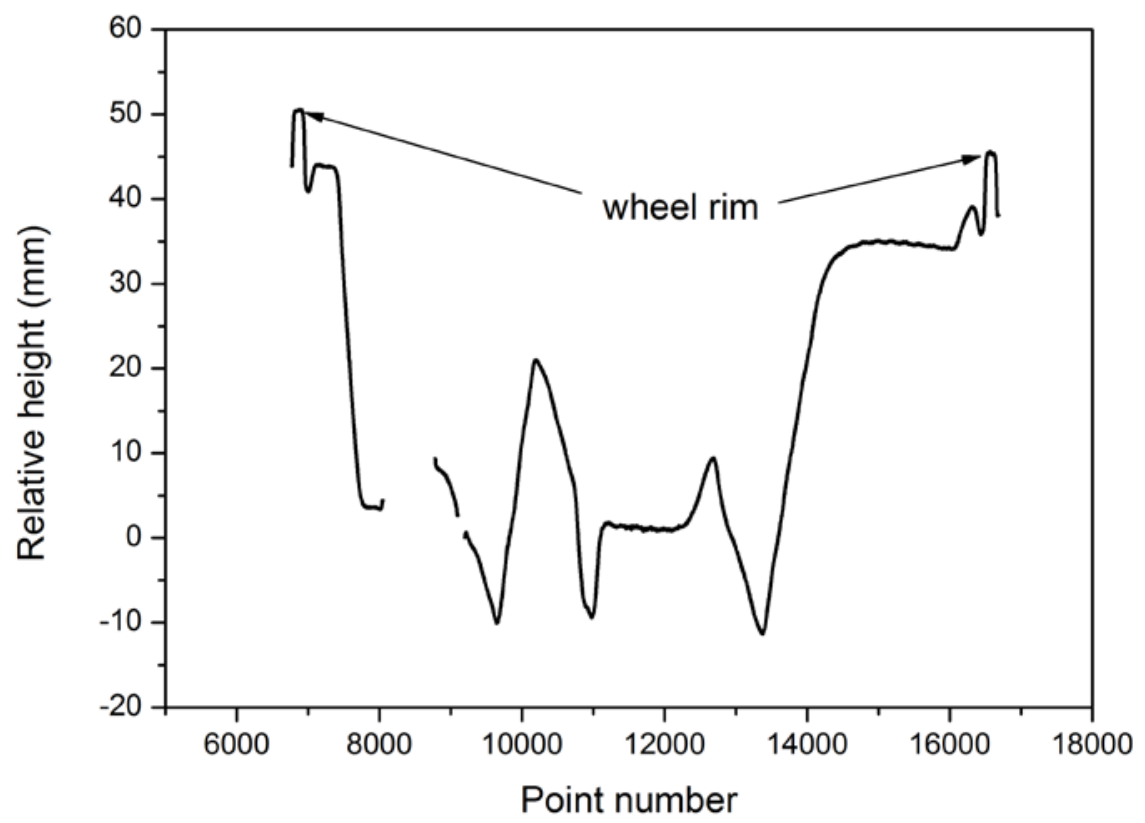

Fig.5 The outline of a wheel scanned by sensor 1

Fig.6 shows the spokes outline scanned by the laser displacement sensor 2. The graph presents the number of points and the relative height of wheel in the same way as shown in the Fig.5. The outline of the spokes is not a straight-forward curve. As the result of the relative height of the sensor 2 , many groups of valid data within the measurement range can be obtained when the laser is scanning the spokes. When the laser scans the gaps between two consecutive spokes, the laser point is out of the measurement range and no valid data can be collected. Therefore, each saltation on the curve corresponds to a spoke, and the number of the wheel spokes can be determined. 


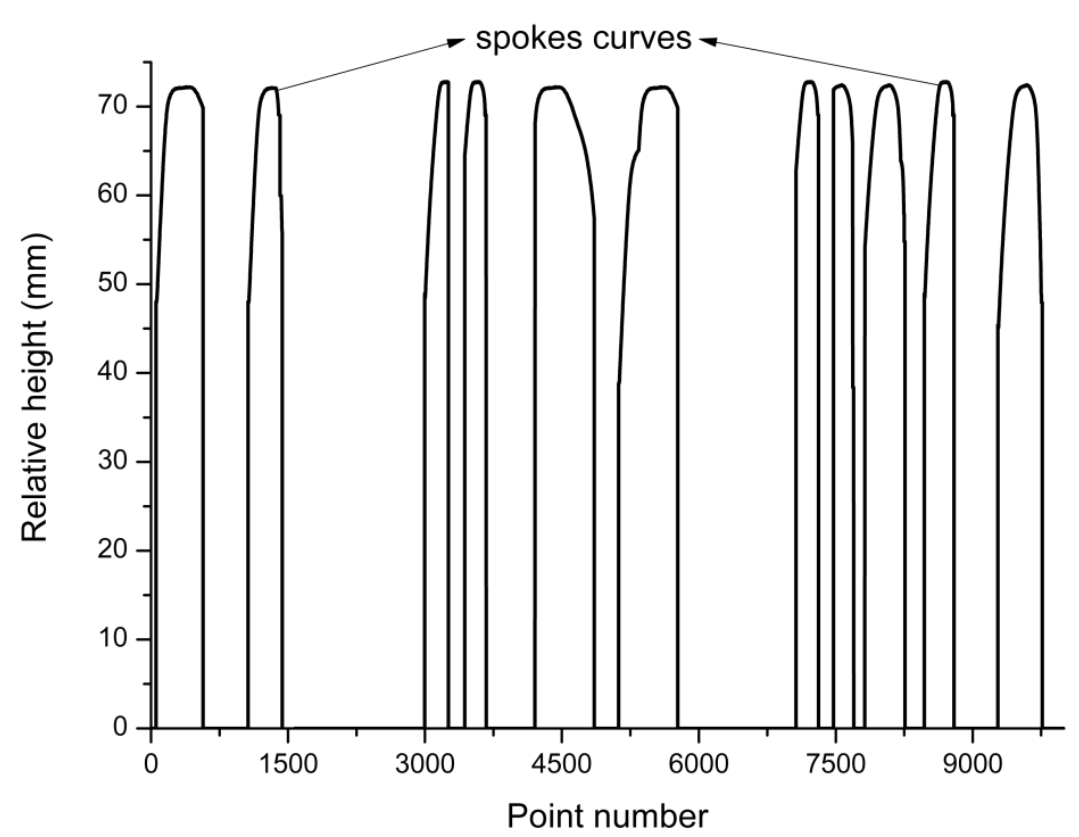

Fig. 6 The outline of spokes scanned by sensor 2

\section{Experiment results}

Ten different types of automobile wheels were recognized by this system. The feature information were determined and compared with those stored in the database, as listed in Table 1.

Table 1 result of 10 wheels identified by the system

\begin{tabular}{|c|c|c|c|c|c|c|c|c|}
\hline \multirow{2}{*}{ Number } & \multicolumn{4}{|c|}{ Identifying information } & \multicolumn{4}{|c|}{ Information in database } \\
\cline { 2 - 9 } & type & $\begin{array}{c}\text { Diameter } \\
(\mathrm{mm})\end{array}$ & $\begin{array}{c}\text { Thickness } \\
(\mathrm{mm})\end{array}$ & $\begin{array}{c}\text { Number } \\
\text { of } \\
\text { spokes }\end{array}$ & $\begin{array}{c}\text { Wheel } \\
\text { type }\end{array}$ & $\begin{array}{c}\text { Diameter } \\
(\mathrm{mm})\end{array}$ & $\begin{array}{c}\text { Thickness } \\
(\mathrm{mm})\end{array}$ & $\begin{array}{c}\text { Number } \\
\text { of } \\
\text { spokes }\end{array}$ \\
\hline 1 & $22 \mathrm{Z} 22$ & 509.5 & 203.9 & 5 & $22 \mathrm{Z} 22$ & $\begin{array}{c}495.3 \sim \\
520.7\end{array}$ & $\begin{array}{c}190.5 \sim \\
215.9\end{array}$ & 5 \\
\hline 2 & $22 \mathrm{Z} 35$ & 508.6 & 216.7 & 15 & $22 \mathrm{Z} 35$ & $\begin{array}{c}495.3 \sim \\
520.7\end{array}$ & $\begin{array}{c}203.2 \sim \\
228.6\end{array}$ & 15 \\
\hline 3 & $22 \mathrm{ZZ7}$ & 457.3 & 205.1 & 15 & $22 \mathrm{Z} 37$ & $\begin{array}{c}444.5 \sim \\
469.9\end{array}$ & $\begin{array}{c}190.5 \sim \\
215.9\end{array}$ & 15 \\
\hline 4 & $22 \mathrm{Z} 43$ & 457.9 & 204.4 & 10 & $22 \mathrm{Z} 43$ & $\begin{array}{c}444.5 \sim \\
469.9\end{array}$ & $\begin{array}{c}190.5 \sim \\
215.9\end{array}$ & 10 \\
\hline 5 & $22 \mathrm{Z} 43$ & 462.1 & 212.9 & 10 & $22 \mathrm{Z} 43$ & $\begin{array}{c}444.5 \sim \\
469.9\end{array}$ & $\begin{array}{c}190.5 \sim \\
215.9\end{array}$ & 10 \\
\hline 6 & $22 \mathrm{Z} 22$ & 510.2 & 203.8 & 5 & $22 \mathrm{Z} 22$ & $\begin{array}{c}495.3 \sim \\
520.7\end{array}$ & $\begin{array}{c}190.5 \sim \\
215.9\end{array}$ & 5 \\
\hline 7 & $22 \mathrm{Z35}$ & 509.3 & 215.2 & 15 & $22 \mathrm{Z35}$ & $\begin{array}{c}495.3 \sim \\
520.7\end{array}$ & $\begin{array}{c}203.2 \sim \\
228.6\end{array}$ & 15 \\
\hline 8 & $22 \mathrm{Z} 43$ & 460.1 & 210.3 & 10 & $22 \mathrm{Z} 43$ & $\begin{array}{c}444.5 \sim \\
469.9\end{array}$ & $\begin{array}{c}190.5 \sim \\
215.9\end{array}$ & 10 \\
\hline 9 & $22 \mathrm{Z} 22$ & 507.9 & 212.1 & 5 & $22 \mathrm{Z} 22$ & $\begin{array}{c}495.3 \sim \\
520.7\end{array}$ & $\begin{array}{c}190.5 \sim \\
215.9\end{array}$ & 5 \\
\hline 10 & $22 \mathrm{Z37}$ & 459.5 & 209.9 & 15 & $22 \mathrm{Z} 37$ & $\begin{array}{c}444.5 \sim \\
469.9\end{array}$ & $\begin{array}{c}190.5 \sim \\
215.9\end{array}$ & 15 \\
\hline
\end{tabular}

According to table 1, all 10 wheels are identified successfully by this system. Although the processing deviation brings inevitable errors for every wheel, the system is intelligent enough to ignore these allowed errors and collects the data to compare with the information in the database completely. 
Results of the automatic wheel recognition system are excellent, and high stability is properly maintained. A data base is established to store normal information of various wheel types, which can be read on the interface of this system. Because of the special soft ware, adding and deleting information can be completed by clicking buttons on the interface. In this way, adding wheel types' data into the database can be performed by plant personnel with little training.

To identify the type of a wheel, the data collected must be compared with feature data stored in the database. Since the soft ware is well-written, it is not necessary to read every data in the database, but only read few of them. In this way, the system minimizes the time spent reading data and reduces the identification time at 20 s per wheel.

\section{Conclusion}

This paper proposes an on-line automatic aluminum wheel recognition system. The system is based on laser trigonometry combined with technologies of optics, mechanics, and electronics. A recognition station, two laser displacement sensors, a ball-screw, an industrial computer with data processing software, and a programmable logic controller (PLC) compose the system. Experiment results show that the system can identify various types of wheels with the total time of 20s per wheel. This system exhibits much better repeatability and reliability compared to the manual performance. In the actual production of aluminum wheel, this system is able to guide and positioning mechanical arms to complete clamping, sorting, moving, and stacking wheels automatically.

\section{Acknowledgements}

The authors wish to acknowledge the financially support by the National High Technology Research and Development Program of China under the Contract of 2013AA031002.

\section{Reference}

[1] Shanfeng Li, Dawei Dong, Lidong Zhu: Application of Alloy Materials in Manufacture of Automobile Wheel, Hot Working Technology, Vol. 43(2014), p. 10-13.

[2] Information on http://www.chinabgao.col.

[3] Qiuhong Yao: Research of One-dimension Laser Triangulation Displacement Measurement Technology, submitted to Harbin Institute of Technology, Haerbin (2014).

[4] Xiaojia Wang, Jun Gao, Lei Wang: Survey on the Laser Triangulation, Chinese Journal of Scientific Instrument, Vol. 25(2004), p. 601-604.

[5] Zhigang Fan: Photoelectric Measurement Technology, edited by Publishing House of Electronics Industry, Beijing (2003).

[6] Slagle J. R, Chang C, Lee R. C: A New Algorithm for Generating Prime Implicates, Computers, IEEE Transactions on, Vol. 19 (1970), p. 304-310. 